assess impairment of function before prescribing melatonin. The main preparation of melatonin prescribed is tablets (94\%), followed by liquid (59\%) then capsules (41\%). Mostly (65\%) slow release medication is given. The minimum dose of melatonin prescribed is $2 \mathrm{mg}$ (range $0.5 \mathrm{mg}-3 \mathrm{mg}$ ), maximum dose range (4-12 mg).

Practice varies in how often children are reviewed. Some (35\%) review in the first 3-4 months, others 6 monthly (29\%). Most (94\%) clinicians offer at least yearly reviews. Routine trials off melatonin are offered by $65 \%$. On average children stay 26 months on melatonin before withdrawal (range 6-120 months). In comments Paediatrician gave views regarding the management of disrupted sleep, the need for good sleep hygiene support, which patient groups melatonin is best suited for and management of melatonin treatment.

Conclusions This survey has highlighted variability amongst Community Paediatricians in the East of England in certain areas of melatonin prescribing, possibly due to lack of uniform standards. With these results we are therefore creating a generic regional algorithm for initiating melatonin in children with disrupted sleep pattern which may form a platform for developing a melatonin prescription and sleep guideline for individual Organisations.

\section{G430(P) IS IT POSSIBLE TO HAVE HIGH STANDARDS PRACTICAL PATHWAY FOR AUTISM SPECTRUM DISORDER?}

A Tarhini. Community Paediatrics, Shropshire Community Health NHS Trust, Shrewsbury, UK

\subsection{6/archdischild-2015-308599.384}

Aims Public awareness of Autism Spectrum Disorder (ASD) is increasing, as well as the demand for assessment. The implementation of high standards and practical pathway for assessment and diagnosis can be a challenge for many trusts including our trust. The aim was to reconstruct the current pathway by the trust to produce a practical pathway for assessment and diagnosis of ASD that meet the high standards of NICE guidelines and following the DSM-V criteria.

Methods Firstly the strengths and weaknesses of the current pathway have been identified. Secondly set up the essential elements of the future pathway defined by DSM-V criteria and NICE guidelines for ASD assessment and diagnosis. An estimated time scale was calculated based on clinic and administration time required.

Results Three essential elements for the assessment have been identified: A) comprehensive meeting with paediatrician, B) multisource observational reports and C) direct assessment. It was possible to incorporate in each step some elements of the NICE guidelines and DSM-V criteria to cover all the essential elements and criteria. Finally, a set of recommendations and suggested pathway for the assessment and diagnosis of ASD was produced. The estimated time to make a definitive outcome about ASD is possible within $240 \mathrm{~min}$ of clinician's direct clinical and administration work.

See Figures 1 and 2

Conclusion It is essential for trusts to have a high standards and practical pathway for ASD diagnosis and assessment in line with NICE guidelines and DSM-V criteria. The current pathway was not satisfactory and a suggested practical pathway has been produced to meet the high standards of practice.

\section{Current Pathway}

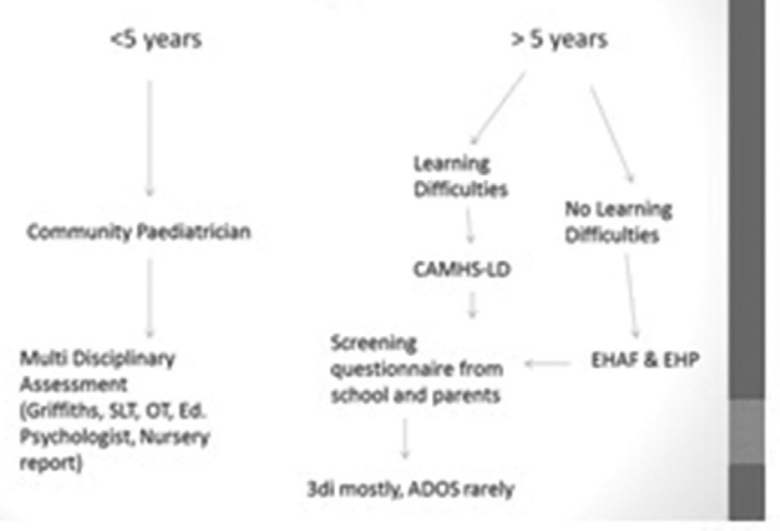

Abstract G430(P) Figure 1 Current pathway

\section{G431(P) AUDIT ON LOOKED AFTER CHILDREN AT RISK OF BLOOD-BORNE INFECTIONS}

P Mikrou, G Cropp, V Sadavarte. Child Development Centre, University Hospital of North Midlands, Stoke-on-Trent, UK

\subsection{6/archdischild-2015-308599.385}

Aims We aimed to assess whether Looked After Children (LAC) at risk of blood-borne infections (BBI) were identified, assessed, tested and referred as per the British Association for Adoption and Fostering (BAAF) guidance, in our specialist LAC service.

Methods Retrospective 12-monthly audit (June13-June14) of all children attending our specialist LAC clinic in Community Paediatrics in our University Teaching Hospital. Data collected from patient health reports, blood investigation results (including maternal antenatal infection screening) and information from Social Care on parental lifestyle. Data analysed using Microsoft Excel.

Results 212 children attended our specialist LAC clinic. 37 children $(17 \%)$ were identified as needing BBI screen. Out of these, only $22(60 \%)$ were screened. 8 out of 22 children $(36 \%)$ had a complete screen (Hepatitis $\mathrm{B} / \mathrm{C}$ and $\mathrm{HIV}$ ) with the remaining having a partial screen. 12 children had Hepatitis $\mathrm{C}$ positive mothers. Worryingly, only 8 of these 12 children (66\%) had BBI screen. There were no Hepatitis B or HIV positive mothers. Reasons for not having BBI screen were difficulties in obtaining consent, failure to identify those children at risk or to get the extended information about parental lifestyle and screening results. BBI screen revealed 2 children positive for Hepatitis C antibodies and appropriate follow-up was arranged. There were no children that had a BBI screen when that was not indicated.

Conclusion Our audit revealed a wide variation in practice as to which children have a BBI screen. We subsequently developed a protocol in the form of two flowcharts. These will be included in the LAC health assessment paperwork and aim to promote clarity and good clinical practice. As failure to obtain consent played an important hindering factor in getting our vulnerable population screened for BBI, we suggested that, when possible, consent is taken at the time of consultation. Improved communication and information sharing between Health and Social Care is essential. Finally, team education is greatly important and will 